RECTANGULAR MIXED FINITE ELEMENTS FOR ELASTICITY

By

Douglas N. Arnold

and

Gerard Awanou

IMA Preprint Series \# 2025

( February 2005)

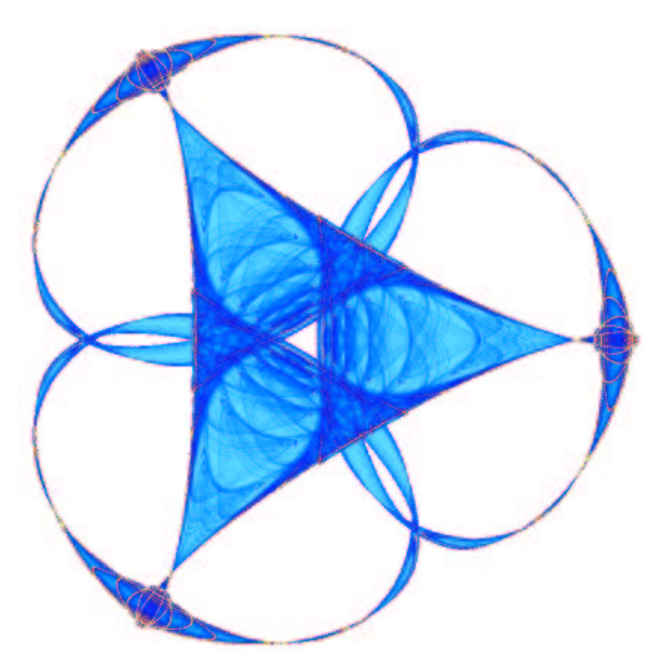

INSTITUTE FOR MATHEMATICS AND ITS APPLICATIONS

UNIVERSITY OF MINNESOTA

514 Vincent Hall

206 Church Street S.E.

Minneapolis, Minnesota 55455-0436

Phone: 612/624-6066 Fax: 612/626-7370

URL: http://www.ima.umn.edu 


\title{
RECTANGULAR MIXED FINITE ELEMENTS FOR ELASTICITY
}

\author{
DOUGLAS N. ARNOLD AND GERARD AWANOU
}

\begin{abstract}
We present a family of stable rectangular mixed finite elements for plane elasticity. Each member of the family consists of a space of piecewise polynomials discretizing the space of symmetric tensors in which the stress field is sought, and another to discretize the space of vector fields in which the displacement is sought. These may be viewed as analogues in the case of rectangular meshes of mixed finite elements recently proposed for triangular meshes. As for the triangular case the elements are closely related to a discrete version of the elasticity differential complex.
\end{abstract}

\section{INTRODUCTION}

Let $\Omega$ be a simply connected polygonal domain of $\mathbb{R}^{2}$, occupied by a linearly elastic body which is clamped on $\partial \Omega$ and let $H(\operatorname{div}, \Omega, \mathbb{S})$ be the space of square-integrable fields taking values in $\mathbb{S}$, the space of symmetric tensors, and which have square integrable divergence. We denote as usual by $L^{2}\left(\Omega, \mathbb{R}^{2}\right)$ the space of square integrable vector fields with values in $\mathbb{R}^{2}$ and $H^{k}(R, X)$ the space of functions with domain $R \subset \mathbb{R}^{2}$, taking values in the finite dimensional space $X$, and with all derivatives of order at most $k$ square integrable. For our purposes, $X$ will be either $\mathbb{S}, \mathbb{R}^{2}$, or $\mathbb{R}$, and in the later case, we will simply write $H^{k}(R)$. The norms in $H^{k}(R, X)$ and $H^{k}(R)$ are denoted respectively $\|\cdot\|_{H^{k}}$ and $\|\cdot\|_{k}$. We also denote by $\mathcal{P}_{k_{1}, k_{2}}(R, X)$ the space of polynomials on $R$ of degree at most $k_{1}$ in $x$ and of degree at most $k_{2}$ in $y$. We write $\mathcal{P}_{k_{1}, k_{2}}$ for $\mathcal{P}_{k_{1}, k_{2}}(R, \mathbb{R})$. For a vector field $v: \Omega \rightarrow \mathbb{R}^{2} \operatorname{grad} v$ is the matrix field with rows the gradient of each component, and $\epsilon(v)=\left[(\operatorname{grad} v)+(\operatorname{grad} v)^{T}\right] / 2$. For a matrix field $\tau$, $\operatorname{div} \tau$ is the vector obtained by applying the divergence operator row-wise and

$$
\sigma: \tau=\sum_{i, j=1}^{2} \sigma_{i j} \tau_{i j} .
$$

The solution $(\sigma, u) \in H(\operatorname{div}, \Omega, \mathbb{S}) \times L^{2}\left(\Omega, \mathbb{R}^{2}\right)$ of the elasticity problem can be characterized as the unique critical point of the Hellinger-Reissner functional

$$
\mathcal{J}(\sigma, v)=\int_{\Omega}\left(\frac{1}{2} A \tau: \tau+\operatorname{div} \tau \cdot v-f \cdot v\right) d x .
$$

The compliance tensor $A=A(x): \mathbb{S} \rightarrow \mathbb{S}$ is given, bounded, and symmetric positive definite uniformly with respect to $x \in \Omega$, and the body force $f$ is also given. The unknowns, $\sigma$ and $u$, represent the the stress and displacement fields, respectively.

A mixed finite element method determines approximate stress field and approximate velocity field $\left(\sigma_{h}, u_{h}\right)$ as the unique critical point of the Hellinger-Reissner functional in a finite

1991 Mathematics Subject Classification. Primary: 65N30, Secondary: 74S05.

Key words and phrases. Mixed method; finite element; elasticity; rectangular.

The work of the first author was supported in part by NSF grant DMS-0411388. 
element space $\Sigma_{h} \times V_{h} \subset H(\operatorname{div}, \Omega, \mathbb{S}) \times L^{2}\left(\Omega, \mathbb{R}^{2}\right)$, where $h$ denotes the mesh size. It can be shown that for a stable approximation, the following two conditions are sufficient: ${ }^{2}$

- $\operatorname{div} \Sigma_{h} \subset V_{h}$.

- There exists a linear operator $\Pi_{h}: H^{1}(\Omega, \mathbb{S}) \rightarrow \Sigma_{h}$, bounded in $\mathcal{L}\left(H^{1}, L^{2}\right)$ uniformly with respect to $h$, and such that $\operatorname{div} \Pi_{h} \sigma=P_{h} \operatorname{div} \sigma$ for all $\sigma \in H^{1}(\Omega, \mathbb{S})$, where $P_{h}: L^{2}\left(\Omega, \mathbb{R}^{2}\right) \rightarrow V_{h}$ denotes the $L^{2}$-projection.

In recent work of Arnold and Winther, ${ }^{2}$ a family of finite element spaces based on triangular meshes was proposed and shown to satisfy these two conditions. In this paper, we will derive an analogous family of finite element spaces based on rectangular meshes and show that they satisfy these two stability conditions.

A key ingredient in the design of mixed finite element methods is the use of differential complexes. ${ }^{1,2}$ In two dimensions, the elasticity complex is

$$
0 \longrightarrow \mathcal{P}_{1}(\Omega) \stackrel{\subset}{\longrightarrow} C^{\infty}(\Omega) \stackrel{J}{\longrightarrow} C^{\infty}(\Omega, \mathbb{S}) \stackrel{\operatorname{div}}{\longrightarrow} C^{\infty}\left(\Omega, \mathbb{R}^{2}\right) \longrightarrow 0,
$$

where $\mathcal{P}_{k}(\Omega)$ is the space of polynomials in two variables of total degree $k$ and $J$, the Airy stress operator defined by

$$
J q:=\left(\begin{array}{cc}
\frac{\partial^{2} q}{\partial y^{2}} & -\frac{\partial^{2} q}{\partial x \partial y} \\
-\frac{\partial^{2} q}{\partial x \partial y} & \frac{\partial^{2} q}{\partial x^{2}}
\end{array}\right)
$$

An analoguous sequence with less smoothness is

$$
0 \longrightarrow \mathcal{P}_{1}(\Omega) \stackrel{\subset}{\longrightarrow} H^{2}(\Omega) \stackrel{J}{\longrightarrow} H(\operatorname{div}, \Omega, \mathbb{S}) \stackrel{\operatorname{div}}{\longrightarrow} L^{2}\left(\Omega, \mathbb{R}^{2}\right) \longrightarrow 0 .
$$

We denote by

$$
\left(\begin{array}{ll}
\mathcal{P}_{k_{1}, k_{2}} & \mathcal{P}_{k_{3}, k_{4}} \\
\mathcal{P}_{k_{3}, k_{4}} & \mathcal{P}_{k_{5}, k_{6}}
\end{array}\right)_{\mathbb{S}}
$$

the space of symmetric matrix fields $\sigma=\left(\begin{array}{ll}\sigma_{11} & \sigma_{12} \\ \sigma_{21} & \sigma_{22}\end{array}\right)$ such that $\sigma_{11} \in \mathcal{P}_{k_{1}, k_{2}}, \sigma_{12}=\sigma_{2,1} \in$ $\mathcal{P}_{k_{3}, k_{4}}$ and $\sigma_{22} \in \mathcal{P}_{k_{5}, k_{6}}$. Similarly, we sometimes write $\left(\begin{array}{l}\mathcal{P}_{k_{1}, k_{2}} \\ \mathcal{P}_{k_{3}, k_{4}}\end{array}\right)$ at the place of $\mathcal{P}_{k_{1}, k_{2}} \times \mathcal{P}_{k_{3}, k_{4}}$. Let $k \geq-2$ be an integer. The following sequence is exact

$$
0 \longrightarrow \mathcal{P}_{1}(\Omega) \stackrel{\subset}{\longrightarrow} \mathcal{P}_{k+4, k+4}(\Omega) \stackrel{J}{\longrightarrow}\left(\begin{array}{ll}
\mathcal{P}_{k+4, k+2} & \mathcal{P}_{k+3, k+3} \\
\mathcal{P}_{k+3, k+3} & \mathcal{P}_{k+2, k+4}
\end{array}\right)_{\mathbb{S}} \stackrel{\text { div }}{\longrightarrow}\left(\begin{array}{l}
\mathcal{P}_{k+3, k+2} \\
\mathcal{P}_{k+2, k+3}
\end{array}\right) \longrightarrow 0 .
$$

To verify the surjectivity of the last map, one can notice that the alternating sum of the dimensions of the spaces in the sequence is zero.

The paper is organized as follows. In the next section, we present our rectangular element in the lowest order case and then show the relation to the elasticity differential complex in the third section. The error estimates are given in the fourth section. In Section 5, we present the higher order elements. Finally we describe a simplified element of low order in the last section. 


\section{The Elements in the Lowest Order Case}

Let $\mathcal{T}_{h}$ denote a conforming partition of $\Omega$ into rectangles of diameter bounded by $h$, which is quasi-uniform in the sense that the aspect ratio of the rectangles is bounded by a fixed constant.

In this section, we define our finite element spaces $\Sigma_{h} \subset H(\operatorname{div}, \mathbb{S})$ and $V_{h} \subset L^{2}(\Omega, \mathbb{R})$ and a bounded operator $\Pi_{h}: H^{1}(\Omega, \mathbb{S}) \rightarrow \Sigma_{h}$ satisfying the commutativity condition

$$
\operatorname{div} \Pi_{h} \tau=P_{h} \operatorname{div} \tau, \quad \tau \in H^{1}(\Omega, \mathbb{S})
$$

and the bound

$$
\left\|\Pi_{h} \tau\right\|_{0} \leq C\|\tau\|_{1}, \quad \tau \in H^{1}(\Omega, \mathbb{S}),
$$

with $c$ independent of $h$. It follows easily that our pair of elements is stable. ${ }^{2}$

We first describe our elements on a single rectangle $R$. We will denote by $n$ the outward normal to an edge of $R$ and define

$$
V_{R}=\left(\begin{array}{l}
\mathcal{P}_{2,1} \\
\mathcal{P}_{1,2}
\end{array}\right), \quad \Sigma_{R}=\left\{\tau \in\left(\begin{array}{ll}
\mathcal{P}_{5,3} & \mathcal{P}_{4,4} \\
\mathcal{P}_{4,4} & \mathcal{P}_{3,5}
\end{array}\right)_{\mathbb{S}} \mid \operatorname{div} \tau \in V_{R}\right\}
$$

The dimension of $V_{R}$ is 12 and the degrees of freedom are given by the values of each component at 6 interior nodes of $R$. The dimension of $\Sigma_{R}$ is at least 45 . To see this, notice that the dimension of the space of matrices with values in $\left(\begin{array}{ll}\mathcal{P}_{5,3} & \mathcal{P}_{4,4} \\ \mathcal{P}_{4,4} & \mathcal{P}_{3,5}\end{array}\right)_{\mathbb{S}}$ is 73 and for those matrix fields, $\operatorname{div} \tau \in \mathcal{P}_{4,3} \times \mathcal{P}_{3,4}$. Since $\operatorname{dim} \mathcal{P}_{4,3}=\operatorname{dim} \mathcal{P}_{4,3}=20$ and $\operatorname{dim} \mathcal{P}_{1,2}=\operatorname{dim} \mathcal{P}_{2,1}=6$, the condition $\operatorname{div} \tau \in V_{R}$ imposes 28 constraints. We will exhibit 45 degrees of freedom after a few preliminaries. This will establish that the dimension of $\Sigma_{R}$ is 45 . (This could also be done with a dimension counting argument.)

The kernel of $\epsilon$ is the space of infinitesimal rigid motions so

$$
\operatorname{dim} \epsilon\left(V_{R}\right)=12-3=9 .
$$

Define

$$
M_{1}(R):=\left\{\tau \in\left(\begin{array}{ll}
\mathcal{P}_{5,3} & \mathcal{P}_{4,4} \\
\mathcal{P}_{4,4} & \mathcal{P}_{3,5}
\end{array}\right)_{\mathbb{S}} \mid \operatorname{div} \tau=0 \text { and } \tau n=0 \text { on } \partial R\right\} .
$$

The following lemma characterizes $M_{1}(R)$.

Lemma 2.1. Let $L_{i}, i=1, \ldots, 4$ be linear functions which define the edges $e_{i}, i=1, \ldots, 4$, of $R$ and define $b_{R}=L_{1} L_{2} L_{3} L_{4}$. We have $J\left(b_{R}^{2} \mathcal{P}_{1,1}\right)=M_{1}(R)$, and so the dimension of $M_{1}(R)$ is 4 .

Proof. First we note that $J\left(b_{R}^{2} \mathcal{P}_{1,1}\right) \subset M_{1}$. Indeed for $q \in \mathcal{P}_{1,1}, b_{R}^{2} q \in \mathcal{P}_{5,5}$ and $\tau=J\left(b_{R}^{2} q\right) \in$ $\left(\begin{array}{ll}\mathcal{P}_{5,3} & \mathcal{P}_{4,4} \\ \mathcal{P}_{4,4} & \mathcal{P}_{3,5}\end{array}\right)$ with $\operatorname{div} \tau=0$. It remains to show that $\tau n=0$ on $\partial R$. This follows from the identities

$$
J(q) n \cdot n=\frac{\partial^{2} q}{\partial s^{2}}, \quad J(q) n \cdot t=\frac{\partial^{2} q}{\partial s \partial n} .
$$

Next, let $v_{i}, i=1, \ldots, 4$, be the vertices of $R$ so that the edge $e_{i}$ is the segment from $v_{i}$ to $v_{i+1}$ with $v_{5} \equiv v_{1}$. Let also $\tau \in M_{1}$. Since $\operatorname{div} \tau=0$, there exists $q \in \mathcal{P}_{5,5}(\Omega)$ such that 
$\tau=J(q)$. Using the identities (2.3) and the vanishing of $\tau n$, we infer that implies that $q$ is linear on each edge and $\partial q / \partial n$ is constant on each edge.

By adding a linear function to $q$, we may assume that $q \equiv 0$ on the edges $e_{1}$ and $e_{4}$ meeting at $v_{1}$. It follows that $q$ and $\nabla q$ vanish at $v_{1}$ and so $q$ and $\partial q / \partial n$ vanish on $e_{1}$. This implies that $L_{1}^{2}$ divides $q$. Similarly $L_{2}^{2}, L_{3}^{2}$, and $L_{4}^{2}$ divide $q$. Thus $q=b_{R}^{2} \tilde{q}$ for some $\tilde{q}$ in $\mathcal{P}_{1,1}$.

We can now give the degrees of freedom for $\Sigma_{R}$.

Lemma 2.2. A matrix field $\tau \in \Sigma_{R}$ is uniquely determined by the following degrees of freedom

(i) the values of each component of $\tau(x)$ at the vertices of $R$ (12 degrees of freedom)

(ii) the first two moments of $(\tau n) \cdot n$ on each edge ( 8 degrees of freedom)

(iii) the first three moments of $(\tau n) \cdot t$ on each edge (12 degrees of freedom)

(iv) the values of $\int_{R} \tau: \phi$ for all $\phi$ in $\epsilon\left(V_{R}\right)$ (9 degrees of freedom)

(v) the values of $\int_{R} \tau: \phi$ for all $\phi$ in $M_{1}(R)$ (4 degrees of freedom)

Proof. We assume that all listed degrees of freedom in the lemma vanish and show that $\tau=0$. Notice that $\tau n \cdot n \in \mathcal{P}_{3}$ and $\tau n \cdot t \in \mathcal{P}_{4}$ on each edge. Since $\tau=0$ at the vertices, the second and third set of degrees of freedom imply that $\tau n=0$ on $\partial R$. Recall that for $v \in H^{1}\left(\Omega, \mathbb{R}^{2}\right)$ and $\tau \in H(\operatorname{div}, \Omega, \mathbb{S})$,

$$
\int_{R}(\operatorname{div} \tau) v d x=-\int_{R} \tau: \epsilon(v) d x+\int_{\partial R} \tau n \cdot v d s .
$$

Since for $\tau \in \Sigma_{R}$, div $\tau \in V_{R}$, using (2.4) for $v \in V_{R}$, we get $\operatorname{div} \tau=0$, i.e., $\tau \in M_{1}(R)$. Finally, using the last set of degrees of freedom, we get $\tau=0$.

We now describe the finite elements on the triangulation $\mathcal{T}_{h}$. We denote by $V_{h}$ the space of vector fields which belong to $V_{R}$ for each $R \in \mathcal{T}_{h}$ and $\Sigma_{h}$ the space of matrix fields which belong piecewise to $\Sigma_{R}$, are continuous at mesh vertices, and have normal components which are continuous across mesh edges.

It remains to define the interpolation operator described at the beginning of this section and to verify the properties (2.1) and (2.2). Because of vertex degrees of freedom, the canonical interpolation operator for $\Sigma_{h}$ is not bounded on $H^{1}(\Omega, \mathbb{S})$, so as in the triangular case, ${ }^{2}$ we consider a family of bounded linear operators $E_{h}^{x}: H^{1}(\Omega, \mathbb{S}) \rightarrow \mathbb{S}$ for each vertex $x$ of the triangulation, and define the interpolation operator $\Pi_{h}: H^{1}(\Omega, \mathbb{S}) \rightarrow \Sigma_{h}$ by

$$
\begin{aligned}
\Pi_{h} \tau(x) & =E_{h}^{x} \tau & & \text { for all vertices } x, \\
\int_{e}\left(\tau-\Pi_{h} \tau\right) n \cdot n v d s & =0 & & \text { for all edges } e \text { and all } v \in \mathcal{P}_{1}(e), \\
\int_{e}\left(\tau-\Pi_{h} \tau\right) n \cdot t v d s & =0 & & \text { for all edges } e \text { and all } v \in \mathcal{P}_{2}(e), \\
\int_{R}\left(\tau-\Pi_{h} \tau\right): \phi d x & =0 & & \text { for all rectangles } R \text { and } \phi \in \epsilon\left(V_{R}\right), \\
\int_{R}\left(\tau-\Pi_{h} \tau\right): \phi d x & =0 & & \text { for all rectangles } R \text { and } \phi \in M_{1}(R) .
\end{aligned}
$$


For $\tau \in H^{1}(\Omega, \mathbb{S}), R \in \mathcal{T}_{h}$ and $v \in V_{R}$, we have

$$
\int_{R}\left(\operatorname{div} \Pi_{h} \tau-\operatorname{div} \tau\right) \cdot v d x=-\int_{R}\left(\Pi_{h} \tau-\tau\right): \epsilon(v) d x+\int_{\partial R}\left(\Pi_{h} \tau-\tau\right) n \cdot v d s .
$$

The first term on the right vanishes because of (2.8). On the other hand, for $v \in V_{R}, v \cdot t \in \mathcal{P}_{2}$ and $v \cdot n \in \mathcal{P}_{1}$ and so the second term also vanishes by (2.6) and (2.7). We conclude that the commutativity condition (2.1) holds. (Note that the proof of (2.1) depended only on the properties (2.6)-(2.8). We need to verify the boundedness condition (2.2). For this, let $\hat{R}=[0,1] \times[0,1]$ be the reference rectangle and let $F: \hat{R} \rightarrow R$ be an affine mapping onto $R, F(\hat{x})=B \hat{x}+b$, with $b=\left(b_{1}, b_{2}\right)$ and

$$
B=\left(\begin{array}{cc}
h_{1} & 0 \\
0 & h_{2}
\end{array}\right)
$$

Although $B$ is symmetric we will write $B^{T}$ so as not to disguise the analogy to more general situations. Given a matrix field $\hat{\tau}: \hat{R} \rightarrow \mathbb{S}$, define $\tau: R \rightarrow \mathbb{S}$ by the matrix Piola transform

$$
\tau(x)=B \hat{\tau}(\hat{x}) B^{T}
$$

We first define an interpolation operator $\Pi_{h}^{0}$ which is local with respect to the triangulation so that its norm can be estimated by scaling arguments. We let $\Pi_{\hat{R}}^{0}: H^{1}(\hat{R}, \mathbb{S}) \rightarrow \Sigma_{\hat{R}}$ by

$$
\begin{aligned}
& \Pi_{\hat{R}}^{0} \hat{\tau}(\hat{x})=0 \quad \text { for all vertices } \hat{x} \text { of } \hat{R} \\
& \int_{\hat{e}}\left(\hat{\tau}-\Pi_{\hat{R}}^{0} \hat{\tau}\right) n \cdot n v d s=0 \quad \text { for all edges } \hat{e} \text { of } \hat{R} \text { and all } v \in \mathcal{P}_{1}(\hat{e}) \\
& \int_{\hat{e}}\left(\hat{\tau}-\Pi_{\hat{R}}^{0} \hat{\tau}\right) n \cdot t v d s=0 \quad \text { for all edges } \hat{e} \text { of } \hat{R} \text { and all } v \in \mathcal{P}_{2}(\hat{e}) \\
& \int_{\hat{R}}\left(\hat{\tau}-\Pi_{\hat{R}}^{0} \hat{\tau}\right): \phi d x=0 \quad \text { for all } \phi \in \epsilon\left(\begin{array}{l}
\mathcal{P}_{2,1} \\
\mathcal{P}_{1,2}
\end{array}\right) \\
& \int_{\hat{R}}\left(\hat{\tau}-\Pi_{\hat{R}}^{0} \hat{\tau}\right): \phi d x=0 \quad \text { for all } \phi \in M_{1}(\hat{R}) \text {. }
\end{aligned}
$$

In view of Lemma $2.2, \Pi_{\hat{R}}^{0}$ is well defined and is easily seen to be bounded. Next define $\Pi_{R}^{0}: H^{1}(R, \mathbb{S}) \rightarrow \Sigma_{R}$ by

$$
\Pi_{R}^{0} \tau(x)=B \Pi_{\hat{R}}^{0} \hat{\tau}(\hat{x}) B^{T}
$$

for each rectangle $R$ of $\mathcal{T}_{h}$ and define $\Pi_{h}^{0}: H^{1}(\Omega, \mathbb{S}) \rightarrow \Sigma_{h}$ by

$$
\left.\left(\Pi_{h}^{0} \tau\right)\right|_{R}=\Pi_{R}^{0} \tau
$$

Note that $\Pi_{h}^{0}$ is bounded on $H^{1}(\Omega, \mathbb{S})$. At the end of this section, we will verify the commutativity property $(2.1)$ with $\Pi_{h}$ replaced by $\Pi_{h}^{0}$. Moreover a standard scaling argument gives

$$
\left\|\Pi_{h}^{0} \tau\right\|_{0} \leq c\left(\|\tau\|_{0}+h\|\tau\|_{1}\right)
$$

where $c$ does not depend on $h$.

Next, let $R_{h}$ be a Clement interpolation operator, ${ }^{3,4}$ which maps $L^{2}(\Omega, \mathbb{R})$ into

$$
\left\{\theta_{h} \in C^{0}(\bar{\Omega}) \mid \theta_{h \mid R} \in \mathcal{P}_{1,1}, \forall R \in \mathcal{T}_{h}\right\}
$$


and denote as well by $R_{h}$ the corresponding operator which maps $L^{2}(\Omega, \mathbb{S})$ into the subspace of $\Sigma_{h}$ of continuous matrix fields whose components are piecewise in $P_{1,1}$. We have

$$
\left\|R_{h} \tau-\tau\right\|_{j} \leq c h^{m-j}\|\tau\|_{m}, \quad 0 \leq j \leq 1, \quad j \leq m \leq 2,
$$

with $c$ independent of $h$. We define our interpolation operator $\Pi_{h}$ by

$$
\Pi_{h}=\Pi_{h}^{0}\left(I-R_{h}\right)+R_{h} .
$$

Then using (2.10) and (2.11), we obtain

$$
\left\|\Pi_{h}^{0}\left(I-R_{h}\right) \tau\right\|_{0} \leq c\left(\left\|\left(I-R_{h}\right) \tau\right\|_{0}+h\left\|\left(I-R_{h}\right) \tau\right\|_{1}\right) \leq c h^{m}\|\tau\|_{m}
$$

for $1 \leq m \leq 2$. It follows that

$$
\left\|\Pi_{h} \tau\right\|_{0} \leq\left\|\Pi_{h}^{0}\left(I-R_{h}\right) \tau\right\|_{0}+\left\|R_{h} \tau\right\|_{0} \leq c\|\tau\|_{1},
$$

i.e., (2.2) holds. Finally, we check the commutativity property (2.1). We have

$$
\begin{aligned}
\operatorname{div} \Pi_{h} & =\operatorname{div} \Pi_{h}^{0}\left(I-R_{h}\right) \tau+\operatorname{div} R_{h} \\
& =\operatorname{div} \Pi_{h}^{0}+\operatorname{div} R_{h}-\operatorname{div} \Pi_{h}^{0} R_{h} \\
& =P_{h} \operatorname{div}+\left(I-P_{h}\right) \operatorname{div} R_{h} \\
& =P_{h} \operatorname{div},
\end{aligned}
$$

where we have used the commutativity property for $\Pi_{h}^{0}$ (which we are about to prove) and $R_{h} \tau \in \Sigma_{h}$.

It remains to establish the commutativity property of $\Pi_{h}^{0}$. For this it is sufficient to verify (2.6)-(2.8) with $\Pi_{h}$ replaced by $\Pi_{h}^{0}$. Notice that for an edge $\hat{e}$ of $\hat{R}$, and the corresponding edge $e$ of $R, n_{\hat{e}}$ is equal to $n_{e}$. Similarly, $\hat{t}=t$. Also $B^{T} n_{e}=\left\|B^{T} n_{e}\right\| n_{\hat{e}}$ as $B \hat{t} \perp n_{e}$. Let $v \in \mathcal{P}_{1}(e)$ and let $\hat{v}(\hat{x})=v(x)$, so $\hat{v} \in \mathcal{P}_{1}(\hat{e})$. Since, $|\hat{e}|=1$, we have

$$
\begin{aligned}
\int_{e} \Pi_{R}^{0} \tau(x) n_{e} \cdot n_{e} v(x) d s & =|e| \int_{\hat{e}} B \Pi_{\hat{R}}^{0} \hat{\tau}(\hat{x}) B^{T} n_{\hat{e}} \cdot n_{\hat{e}} \hat{v}(\hat{x}) d \hat{s} \\
& =|e| B \int_{\hat{e}} \Pi_{\hat{R}}^{0} \hat{\tau}(\hat{x}) B^{T} n_{\hat{e}} \cdot n_{\hat{e}} \hat{v}(\hat{x}) d \hat{s} \\
& =|e|\left\|B^{T} n_{e}\right\| B \int_{\hat{e}} \Pi_{\hat{R}}^{0} \hat{\tau}(\hat{x}) n_{\hat{e}} \cdot n_{\hat{e}} \hat{v}(\hat{x}) d \hat{s} \\
& =|e|\left\|B^{T} n_{e}\right\| B \int_{\hat{e}} \hat{\tau}(\hat{x}) n_{\hat{e}} \cdot n_{\hat{e}} \hat{v}(\hat{x}) d \hat{s} \\
& =|e| \int_{\hat{e}} B \hat{\tau}(\hat{x}) B^{T} n_{\hat{e}} \cdot n_{\hat{e}} \hat{v}(\hat{x}) d \hat{s} \\
& =\int_{e} \tau(x) n_{e} \cdot n_{e} v(x) d s
\end{aligned}
$$

verifying (2.6). Similarly, for $v \in \mathcal{P}_{2}(e)$,

$$
\int_{e} \Pi_{R}^{0} \tau(x) n_{e} \cdot t_{e} v(x) d s=\int_{e} \tau(x) n_{e} \cdot t_{e} v(x) d s
$$


verifying (2.7). To verify (2.8), we infer from (2.6) and (2.7) that

$$
\int_{\partial R} \Pi_{R}^{0} \tau(x) n \cdot v(x) d s=\int_{\partial R} \tau(x) n \cdot v(x) d s
$$

for all $v \in \mathcal{P}_{2,1} \times \mathcal{P}_{1,2}$. Next notice that since $\Pi_{\hat{R}}^{0}$ satisfies $(2.6)-(2.8)$, we have for $\hat{v} \in$ $P_{2,1} \times P_{1,2}$,

$$
\begin{aligned}
\int_{\hat{R}} \operatorname{div} \hat{\tau} \hat{v}(\hat{x}) d \hat{x} & =-\int_{\hat{R}} \hat{\tau}: \epsilon(\hat{v}) d \hat{x}+\int_{\partial \hat{R}} \hat{\tau} \hat{n} \cdot \hat{v} d \hat{s} \\
& =-\int_{\hat{R}} \Pi_{\hat{R}}^{0} \hat{\tau}: \epsilon(\hat{v}) d \hat{x}+\int_{\partial \hat{R}} \Pi_{\hat{R}}^{0} \hat{\tau} \hat{n} \cdot \hat{v} d \hat{s} \\
& =\int_{\hat{R}} \operatorname{div} \Pi_{\hat{R}}^{0} \hat{\tau} \hat{v}(\hat{x}) d \hat{x}
\end{aligned}
$$

Next, a direct computation shows that for $\tau(x)=B \hat{\tau}(\hat{x}) B^{T}$,

$$
\operatorname{div} \tau=B \operatorname{div} \hat{\tau}
$$

We therefore have

$$
\begin{aligned}
\int_{R}\left(\operatorname{div} \tau-\operatorname{div} \Pi_{h}^{0} \tau\right) \cdot v d x & =(\operatorname{det} B) B \int_{\hat{R}} \operatorname{div}\left(\hat{\tau}-\Pi_{\hat{R}}^{0} \hat{\tau}\right) \cdot \hat{v} d \hat{x} \\
& =(\operatorname{det} B) \int_{\hat{R}} \operatorname{div}\left(\hat{\tau}-\Pi_{\hat{R}}^{0} \hat{\tau}\right) \cdot\left(B^{T} \hat{v}\right) d \hat{x}=0
\end{aligned}
$$

where we have noted that $B^{T} \hat{v} \in P_{2,1} \times P_{1,2}$ and applied (2.15) at the last step. Now, for $v \in P_{2,1} \times P_{1,2}$,

$$
\begin{aligned}
\int_{R} \Pi_{h}^{0} \tau(x): & \epsilon(v)(x) d x=-\int_{R} \operatorname{div} \Pi_{h}^{0} \tau(x) \cdot v(x) d x+\int_{\partial R} \Pi_{h}^{0} \tau(x) n \cdot v(x) d s \\
= & -\int_{R} \operatorname{div} \Pi_{h}^{0} \tau(x) \cdot v(x) d x+\int_{\partial R} \tau(x) n \cdot v(x) d s \\
& =\int_{R} \tau(x): \epsilon(v(x)) d x+\int_{R} \operatorname{div} \tau(x) \cdot v(x) d x-\int_{R} \operatorname{div} \Pi_{h}^{0} \tau(x) \cdot v(x) d x \\
& =\int_{R} \tau(x): \epsilon(v)(x) d x,
\end{aligned}
$$

where we have used (2.14) in the second step and (2.16) in the last. This completes the proof.

\section{Discrete Version of the Elasticity Sequence}

The element described in this paper was discovered by looking for a discrete version of (1.1). The starting point is the construction of an $H^{2}$ element which we now describe first on a single rectangle.

We take $Q_{R}=\mathcal{P}_{5,5}(R)$ with the following 36 degrees of freedom

(i) derivatives up to order 2 at each vertex $(6 \times 4=24$ degrees of freedom $)$

(ii) moments of degree 0 and 1 of $\partial q / \partial n$ on each edge $(2 \times 4=8$ degrees of freedom)

(iii) $\int_{R} J(q): \phi d x$ for all $\phi \in M_{1}$ (4 degrees of freedom) 
We next show that we have described a unisolvent set of degrees of freedom. For this we assume that all degrees of freedom of $Q_{R}$ vanish. On each edge, $q$ is a polynomial of degree 5 with triple roots at each vertex. Therefore $q \equiv 0$ on each edge and we can write $q=\bar{q} L_{1} L_{2} L_{3} L_{4}$, with $\bar{q} \in \mathcal{P}_{3,3}$. We have

$$
\left.\frac{\partial q}{\partial n_{1}}\right|_{e_{1}}=\bar{q} \frac{\partial L_{1}}{\partial n_{1}} L_{2} L_{3} L_{4}
$$

so that $q_{1}:=\partial q / \partial n_{1} \in \mathcal{P}_{5}\left(e_{1}\right)$ on the edge $e_{1}$; (this can also be seen easily on the reference rectangle). Clearly, $q_{1}$ and $\partial q_{1} / \partial s$ are zero at the vertices of $e_{1}$, so using the second set of degrees of freedom

$$
0=\int_{e_{1}} q_{1} \frac{\partial^{4} q_{1}}{\partial s^{4}}=-\int_{e_{1}} \frac{\partial q_{1}}{\partial s} \frac{\partial^{3} q_{1}}{\partial s^{3}}=\int_{e_{1}} \frac{\partial^{2} q_{1}}{\partial s^{2}} \frac{\partial^{2} q_{1}}{\partial s^{2}}
$$

We conclude that $\partial^{2} q_{1} / \partial s^{2}=0$ on $e_{1}$, so that $q_{1}=0$ on $e_{1}$ and therefore $\bar{q}=0$ on $e_{1}$. Since a similar argument show that $\bar{q}$ vanishes on each edge, we have $q=\tilde{q} b_{R}^{2}$ with $\tilde{q} \in \mathcal{P}_{1,1}$. Taking $\phi=J\left(b_{R}^{2} \tilde{q}\right)$ in the last set of degrees of freedom, we get $q=0$, since the kernel of $J$ is $\mathcal{P}_{1}(R)$.

The finite element $Q_{h} \subset H^{2}(\Omega)$ is assembled the usual way and we define an interpolation operator $I_{h}: C^{\infty}(\Omega) \rightarrow Q_{h}$ by requiring at each vertex $x$

$$
\begin{aligned}
I_{h} q(x) & =q(x), \\
\left(\nabla I_{h} q\right)(x) & =(\nabla q)(x), \\
\left(J I_{h} q\right)(x) & =E_{h}^{x}(J q)(x),
\end{aligned}
$$

and

$$
\begin{aligned}
\int_{e} \frac{\partial I_{h} q}{\partial n}(s) f d s & =\int_{e} \frac{\partial q}{\partial n}(s) f d s \quad \text { for all edges and all } f \in \mathcal{P}_{1}(e) \\
\int_{R} J\left(I_{h} q\right): \phi d x & =\int_{R} J(q): \phi d x \quad \text { for all } \phi \in M_{1}
\end{aligned}
$$

We can now describe our discrete version of the elasticity differential complex:

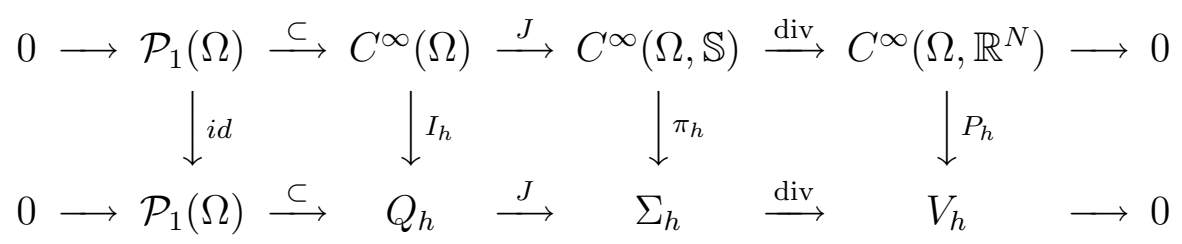

We only need to check the commutativity property $\Pi_{h} J q=J I_{h} q$, i.e. $\Pi_{h} \tau=\sigma$ with $\tau=J(q)$ and $\sigma=J I_{h} q$.

We have by definition of $I_{h},\left(J I_{h} q\right)(x)=\left(E_{h}^{x} J q\right)(x)$. Recall that for $\tau=J q, \partial^{2} q / \partial s^{2}=$ $\tau n \cdot n$ and $\partial^{2} q /(\partial s \partial n)=\tau n \cdot t$. Now, for $v \in \mathcal{P}_{1}(e)$, using (3.1) and (3.2) after a double integration by parts

$$
\int_{e}\left(J I_{h} q-J q\right) n \cdot n v d s=\int_{e} \frac{\partial^{2}\left(I_{h} q-q\right)}{\partial s^{2}} v d s=0 .
$$

By (3.2) and (3.4), we also have for $v \in \mathcal{P}_{2}(e)$

$$
\int_{e}\left(J I_{h} q-J q\right) n \cdot t v d s=\int_{e} \frac{\partial^{2}\left(I_{h} q-q\right)}{\partial s \partial n} v d s=-\int_{e} \frac{\partial\left(I_{h} q-q\right)}{\partial n} \frac{\partial v}{\partial s} d s=0 .
$$


Next, for $\phi=\epsilon(v) \in \epsilon\left(V_{R}\right)$, using (2.4)

$$
\begin{aligned}
\int_{R}\left(J I_{h} q-J q\right): \phi & =\int_{\partial R}\left(J I_{h} q-J q\right) n \cdot v d s \\
& =0
\end{aligned}
$$

by the above identities since for $v \in V_{R}, v \cdot t \in \mathcal{P}_{2}(e)$ and $v \cdot n \in \mathcal{P}_{1}(e)$ for each edge $e$.

Finally, for $\phi \in M_{1}(R)$,

$$
\int_{R}\left(J I_{h} q-J q\right): \phi=0
$$

by $(3.5)$.

\section{Error Analysis}

Since our pair of elements is stable, we have from the theory of mixed methods the following quasioptimal estimate

$$
\left\|\sigma-\sigma_{h}\right\|_{H(\operatorname{div})}+\left\|u-u_{h}\right\|_{L^{2}} \leq c \inf _{\tau \in \Sigma_{h}, v \in V_{h}}\left(\|\sigma-\tau\|_{H(\text { div })}+\|u-v\|_{L^{2}}\right) .
$$

This gives an $\mathrm{O}\left(h^{2}\right)$ error estimate for smooth solutions. It can be refined in various ways. For the interpolation operator $\Pi_{h}$, c.f. (2.12), we have

$$
I-\Pi_{h}=\left(I-R_{h}\right)-\Pi_{h}^{0}\left(I-R_{h}\right)
$$

so using (2.11) and (2.13), we get

$$
\left\|\Pi_{h} \tau-\tau\right\|_{0} \leq c h^{m}\|\tau\|_{m}, \quad 1 \leq m \leq 2 .
$$

We also recall that the projection operator $P_{h}$ satisfies the error estimate

$$
\left\|P_{h} v-v\right\|_{0} \leq c h^{m}\|v\|_{m}, \quad 0 \leq m \leq 2
$$

since $V_{R}$ contains the vector fields with components in $P_{1,1}$. We then have the following result, whose proof is similar to the one in Arnold-Winther. ${ }^{2}$

Theorem 4.1. Let $(\sigma, u)$ denote the unique critical point of the Hellinger-Reissner functional over $H(\operatorname{div}, \Omega, \mathbb{S}) \times L^{2}\left(\Omega, \mathbb{R}^{2}\right)$ and let $\left(\sigma_{h}, u_{h}\right)$ be the unique critical point over $\Sigma_{h} \times V_{h}$. Then

$$
\begin{aligned}
\left\|\sigma-\sigma_{h}\right\|_{0} & \leq c h^{m}\|\sigma\|_{m}, \quad 1 \leq m \leq 2, \\
\left\|\operatorname{div} \sigma-\operatorname{div} \sigma_{h}\right\|_{0} & \leq c h^{m}\|\operatorname{div} \sigma\|_{m}, \quad 0 \leq m \leq 2, \\
\left\|u-u_{h}\right\|_{0} \leq c h^{m}\|u\|_{m+1}, & 1 \leq m \leq 2
\end{aligned}
$$

\section{Higher Order Elements}

In this section we describe a family of stable element pairs, one for each degree $k \geq 1$. The case $k=1$ is the one treated above. We first describe the elements on a single rectangle:

$$
V_{R}=\left(\begin{array}{l}
\mathcal{P}_{k+1, k} \\
\mathcal{P}_{k, k+1}
\end{array}\right), \quad \Sigma_{R}=\left\{\tau \in\left(\begin{array}{ll}
\mathcal{P}_{k+4, k+2} & \mathcal{P}_{k+3, k+3} \\
\mathcal{P}_{k+3, k+3} & \mathcal{P}_{k+2, k+4}
\end{array}\right)_{\mathbb{S}} \mid \operatorname{div} \tau \in V_{R}\right\} .
$$


We have $\operatorname{dim} V_{R}=2(k+1)(k+2)$ and the degrees of freedom are given by the values of each component at $(k+1)(k+2)$ interior nodes of $R$.

$$
\begin{aligned}
\operatorname{dim} \Sigma_{R} \geq d_{k} & :=2 \operatorname{dim} \mathcal{P}_{k+4, k+2}+\operatorname{dim} \mathcal{P}_{k+3, k+3}-2\left(\operatorname{dim} \mathcal{P}_{k+3, k+2}-\operatorname{dim} \mathcal{P}_{k+1, k}\right) \\
& =2(k+3)(k+5)+(k+4)^{2}-2[(k+3)(k+4)-(k+1)(k+2)] \\
& =3 k^{2}+16 k+26 .
\end{aligned}
$$

As in the lowest order case, let us define

$$
M_{k}(R):=\left\{\tau \in\left(\begin{array}{ll}
\mathcal{P}_{k+4, k+2} & \mathcal{P}_{k+3, k+3} \\
\mathcal{P}_{k+3, k+3} & \mathcal{P}_{k+2, k+4}
\end{array}\right)_{\mathbb{S}} \mid \operatorname{div} \tau=0, \quad \tau n=0 \text { on } \partial R\right\} .
$$

Similar arguments to Lemma 2.1 show that for $\tau$ in $M_{k}(R), \tau=J\left(b_{R}^{2} q\right)$ for some $q \in \mathcal{P}_{k, k}$ with $b_{R}=L_{1} L_{2} L_{3} L_{4}$, hence $\operatorname{dim} M_{k}(R)=(k+1)^{2}$. Next, the dimension of $\epsilon\left(V_{R}\right)$ is $2(k+1)(k+2)-3=2 k^{2}+6 k+1$ and $\epsilon\left(V_{R}\right)$ is orthogonal to $M_{k}(R)$ by $(2.4)$. We conclude that the dimension of

$$
N_{k}(R)=\epsilon\left(V_{R}\right)+M_{k}(R)
$$

is $3 k^{2}+8 k+2$. Notice also that for $\tau \in\left(\begin{array}{ll}\mathcal{P}_{k+4, k+2} & \mathcal{P}_{k+3, k+3} \\ \mathcal{P}_{k+3, k+3} & \mathcal{P}_{k+2, k+4}\end{array}\right)_{\mathbb{S}}, \tau n \cdot n \in \mathcal{P}_{k+2}$ and $\tau n \cdot t \in \mathcal{P}_{k+3}$. We can now give the degrees of freedom of $\Sigma_{R}$ :

(i) the values of each component of $\tau$ at each vertex of $R,(3 \times 4=12$ degrees of freedom $)$

(ii) the moments of degree at most $k$ of $\tau n \cdot n$ on each edge $(4(k+1)$ degrees of freedom)

(iii) the moments of degree at most $k+1$ of $\tau n \cdot t$ on each edge $(4(k+2)$ degrees of freedom)

(iv) the values of $\int_{R} \tau: \phi$ for all $\phi \in N_{k}(R)$.

The proof of unisolvency is identical to the lowest order case. The corresponding error estimates are similar to those in the triangular case. ${ }^{2}$ We recall that the Clement interpolant which takes values into the space of continuous matrix fields with components in $\mathcal{P}_{k, k}$ satisfies the error estimate

$$
\left\|R_{h} v-v\right\|_{j} \leq c h^{m-j}\|v\|_{m}, \quad 0 \leq j \leq 1, j \leq m \leq k+1
$$

and the projection operator into $V_{R}$ satisfies

$$
\left\|P_{h}^{k} v-v\right\|_{0} \leq c h^{m}\|v\|_{m}, \quad 0 \leq m \leq k+1 .
$$

We have

Theorem 5.1. Let $(\sigma, u)$ denote the unique critical point of the Hellinger-Reissner funtional over $H(\operatorname{div}, \Omega, \mathbb{S}) \times L^{2}\left(\Omega, \mathbb{R}^{2}\right)$ and let $\left(\sigma_{h}, u_{h}\right)$ be the unique critical point over $\Sigma_{h} \times V_{h}$. Then

$$
\begin{gathered}
\left\|\sigma-\sigma_{h}\right\|_{0} \leq c h^{m}\|\sigma\|_{m}, \quad 1 \leq m \leq k+1, \\
\left\|\operatorname{div} \sigma-\operatorname{div} \sigma_{h}\right\|_{0} \leq c h^{m}\|\operatorname{div} \sigma\|_{m}, \quad 0 \leq m \leq k+1, \\
\left\|u-u_{h}\right\|_{0} \leq c h^{m}\|u\|_{m+1}, \quad 1 \leq m \leq k+1 .
\end{gathered}
$$




\section{A Simplified Element of Low Order}

Let $R M(R)$ be the space of infinitesimal rigid motions on $R$, i.e., vector fields of the form $\left(a-c x_{2}, b-c x_{1}\right)$. We take $V_{R}=R M(R)$ and

$$
\Sigma_{R}=\left\{\tau \in\left(\begin{array}{ll}
\mathcal{P}_{5,3} & \mathcal{P}_{4,4} \\
\mathcal{P}_{4,4} & \mathcal{P}_{3,5}
\end{array}\right)_{\mathbb{S}} \text { and } \operatorname{div} \tau \in R M(R)\right\}
$$

Then, $\operatorname{dim} \Sigma_{R}=36$ since the condition div $\tau \in R M(R)$ imposes $40-3=37$ conditions on $\tau$. We take as degrees of freedom

(i) the values of each component of $\tau(x)$ at the vertices of $R$ (12 degrees of freedom)

(ii) the first two moments of $(\tau n) \cdot n$ on each edge ( 8 degrees of freedom)

(iii) the first three moments of $(\tau n) \cdot t$ on each edge (12 degrees of freedom)

(iv) the values of $\int_{R} \tau: \phi$ for all $\phi$ in

$$
M_{1}(R):=\left\{\tau \in\left(\begin{array}{ll}
\mathcal{P}_{5,3} & \mathcal{P}_{4,4} \\
\mathcal{P}_{4,4} & \mathcal{P}_{3,5}
\end{array}\right)_{\mathbb{S}} \mid \operatorname{div} \tau=0, \quad \tau n=0 \text { on } \partial R\right\}
$$

\section{(4 degrees of freedom)}

The proof of unisolvency is as in the lowest order case, since for $v \in R M(R), \epsilon(v)=0$ and again the error estimates are similar to those of the triangular case since $\Sigma_{R}$ contains the matrix fields with components in $\mathcal{P}_{0,0}$ and $V_{R}$ contains the vector fields with components in $\mathcal{P}_{0,0}$.

$$
\begin{gathered}
\left\|\sigma-\sigma_{h}\right\|_{0} \leq \operatorname{ch}\|\sigma\|_{1} \\
\left\|\operatorname{div} \sigma-\operatorname{div} \sigma_{h}\right\|_{0} \leq h^{m}\|\operatorname{div} \sigma\|_{m}, \quad 0 \leq m \leq 1, \\
\left\|u-u_{h}\right\|_{0} \leq c h\|u\|_{2} .
\end{gathered}
$$

\section{REFERENCES}

[1] D. Arnold, Differential complexes and numerical stability, in Proceedings of the International Congress of Mathematicians, Vol. I: Plenary Lectures and Ceremonies, L. Tatsien, ed. (Higher Education Press, Beijing, 2002), pp. 137-157.

[2] D. Arnold and R. Winther, Mixed finite elements for elasticity, Numer. Math. 92 (2002) 401-419.

[3] P. Clément, Approximation by finite element functions using local regularization, Rev. Française Automat. Informat. Recherche Opérationnelle Sér. Rouge 9 (1975), 77-84.

[4] G. Bernardi and V. Girault, A local regularization operator for triangular and quadrilateral finite elements, SIAM J. Numer. Anal. 35 (1998) 1893-1916.

Institute for Mathematics and its Applications, University of Minnesota, 400 Lind Hall, 207 Church Street S.E., Minneapolis, MN, 55455, USA

E-mail address: arnold@ima.umn.edu

$U R L:$ http://www.ima.umn.edu/ arnold

E-mail address: awanou@ima.umn.edu

$U R L:$ http://www.ima.umn.edu/ ^awanou 